\title{
A Mimic of Hepatic Encephalopathy: Two Cases of Cryptococcal Meningitis in North America
}

\author{
Peng-Sheng Ting* ${ }^{1}$, Anant Agarwalla ${ }^{2}$ and Tinsay A. Woreta ${ }^{2}$ \\ ${ }^{1}$ School of Medicine, The Johns Hopkins Hospital, Baltimore, MD, USA; ${ }^{2}$ Division of Gastroenterology/Hepatology, The Johns \\ Hopkins Hospital, Baltimore, MD, USA
}

\begin{abstract}
In the non-human immunodeficiency virus infected population, cryptococcosis occurs primarily in people who are functionally immunosuppressed, including patients who have undergone solid organ transplantation requiring immunosuppressive medications, are on corticosteroids, or have renal failure or cirrhosis. Cryptococcal meningitis poses a particular challenge in the setting of cirrhosis because its clinical presentation can mimic hepatic encephalopathy. Here, we describe two patients with decompensated cirrhosis, both with a known history of hepatic encephalopathy who had lumbar punctures and were found to have cryptococcal meningitis. The first patient had a subacute fluctuating change in mental status, while the second patient had progressive subacute headaches, gait disturbance, and hearing loss. Both patients were treated with amphotericin B and flucytosine induction, but only the second survived to maintenance therapy. These cases demonstrate the importance of having a high index of suspicion for cryptococcal meningitis in cirrhosis and having a low threshold for performing a lumbar puncture when altered mental status or other neurologic complaints are not fully explained by hepatic encephalopathy. We also provide a brief review of the pathobiology of cryptococcal infection in cirrhosis and highlight the challenges in therapy.
\end{abstract}

Citation of this article: Ting PS, Agarwalla A, Woreta TA. A mimic of hepatic encephalopathy: Two cases of cryptococcal meningitis in North America. J Clin Transl Hepatol 2019;7 (2):191-193. doi: 10.14218/JCTH.2019.00005.

\section{Introduction}

In patients with cirrhosis, neurologic complaints are frequently seen in the setting of hepatic encephalopathy (HE), and clinicians are often reassured once patients show signs of improvement in mental status upon treatment for HE. Cryptococcal meningitis poses a particular challenge in the setting of cirrhosis because its clinical presentation can mimic HE. Most of the data for the description of and treatment for cryptococcosis has come from studies of the human immunodeficiency virus (HIV)-infected population, who accounted for more than

Keywords: Cryptococcal meningitis; Decompensated cirrhosis; Liver transplant. Abbreviations: CSF, cerebrospinal fluid; $\mathrm{HE}$, hepatic encephalopathy; HIV, human immunodeficiency virus; LP, lumbar puncture; MELD, model for endstage liver disease.

Received: 24 January 2019; Revised: 6 March 2019; Accepted: 22 March 2019 *Correspondence to: Peng-Sheng Ting, School of Medicine, The Johns Hopkins Hospital, Baltimore 21287, MD, USA. Tel: +1-646-407-6759, E-mail: ting.pengsheng@ gmail.com
$80 \%$ of incident cryptococcosis cases occurring prior to widespread availability of antiretroviral therapy. In the era of antiretroviral therapy, however, we are beginning to see a larger proportion of cryptococcal infections in non-HIV infected patients, and up to $36 \%$ of these patients have cirrhosis. ${ }^{1-4}$

In the non-HIV infected population, cryptococcal infection is commonly seen in functionally immunosuppressed patients, including patients who have undergone solid organ transplantation requiring immunosuppressive medications, are on corticosteroids, or have renal failure or cirrhosis. ${ }^{5}$ Cryptococcal meningitis typically manifests as a subacute to chronic meningoencephalitis, with gradually worsening headaches and common symptoms such as nausea, vomiting, lethargy, personality changes, and confusion. Unlike in the population with acquired immune deficiency syndrome (commonly known as AIDS), wherein cryptococcal meningitis is often promptly suspected and disseminates to other organs if untreated, cryptococcal meningitis in cirrhotic patients often has a more insidious presentation. ${ }^{5}$ The frequently observed lag time to diagnosis makes cryptococcal meningitis an often fatal disease in cirrhosis. ${ }^{4,6}$ If the diagnosis is not made early or missed prior to liver transplantation, the outcome can be devastating.

\section{Pathobiology}

Cryptococcal infections in humans are due to Cryptococcus neoformans and, much less frequently, Cryptococcus gatti. ${ }^{7}$ Cryptococcus species are ubiquitous in the environment and are easily aerosolized and inhaled. ${ }^{8}$ Once in the lungs, the primary host responses are complement-mediated macrophage and neutrophil phagocytosis and intracellular killing and natural killer cell response. The ensuing $T$ cell immunity ensures Cryptococcus is either eradicated or confined in walled-off granulomas. Cryptococcus species avoid phagocytosis and killing by a variety of mechanisms, most important among them being the polysaccharide capsule that resists opsonization and melanin production, ultimately thwarting intracellular killing by resisting oxidative stress. ${ }^{9-11}$

If the organism is permitted to replicate to a critical mass due to a deficiency in the aforementioned immunologic mechanisms, it can disseminate hematogenously to the central nervous system either by a "Trojan Horse" method (piggybacking in macrophages) or by direct transcellular entry through the cerebral capillaries. ${ }^{12}$ In cirrhosis with synthetic dysfunction, there is a marked decrease in complement and immunoglobulin production, leading to a decrease in both innate and humoral immunity. Furthermore, the liver is a reservoir for both $B$ and $T$ cells and T cell immune deficiencies have also been described in cirrhosis. ${ }^{13,14}$ Though no one specific immune deficiency in 
cirrhosis has been identified as predisposing to cryptococcal meningitis, the organism likely reactivates from the lung following worsened innate, complement-mediated, and Tcell immune function with progressive liver disease.

\section{Case 1}

A 69 year-old woman presented with worsening confusion and agitation over the span of a month. She had a past medical history of decompensated cirrhosis due to hepatitis $C$ virus complicated by $\mathrm{HE}$, ascites, and untreated hepatocellular carcinoma. History regarding her illness was obtained primarily from her family and was limited, particularly regarding lactulose compliance, frequency of bowel movements, and symptoms of infection. Her daughter did report the patient had altered sleeping habits and exhibited bizarre behaviors, such as leaving the house not fully dressed. The patient's neurologic exam on admission was notable for being oriented only to self intermittently, without asterixis or any focal neurologic deficits. Laboratory values on admission were notable for a model for end-stage liver disease (commonly known as MELD) score of 17 , total bilirubin of $4 \mathrm{mg} / \mathrm{dL}$, international normalized ratio of 1.6 , creatinine of 0.8 , albumin of $2 \mathrm{~g} / \mathrm{dL}$, venous ammonia of $34 \mu \mathrm{mol} / \mathrm{L}$ (reference range: $0-32 \mu \mathrm{mol} / \mathrm{L}$ ), venous blood gas with a pH of 7.39 and $\mathrm{pCO}_{2}$ of 42 , white blood cell count of $4,080 / \mathrm{mm}^{3}$, hemoglobin of $8.8 \mathrm{~g} / \mathrm{dL}$, platelet count of 64,000 / $\mathrm{mm}^{3}$, negativity for pyuria on urinalysis and urine toxicology, and normal electrolytes and thyroid studies.

The patient's mental status did not improve after receiving treatment for HE with lactulose and rifaximin therapy following admission. MRI of the brain (Fig. 1) was suggestive of meningitis or cerebritis.

Lumbar puncture (LP) was performed and cerebrospinal fluid (CSF) analysis showed white blood cell count of $99 / \mathrm{mm}^{3}$ (61 monocytes, 38 neutrophils), glucose of $33 \mathrm{mg} / \mathrm{dL}$, protein of $188.8 \mathrm{mg} / \mathrm{dL}$, reactivity for cryptococcal antigen, and positivity for Cryptococcus neoformans in fungal culture. First opening pressure was $33 \mathrm{cmH}_{2} \mathrm{O}$ and subsequent opening pressures remained above $30 \mathrm{cmH}_{2} \mathrm{O}$, necessitating the insertion of a lumbar drain during the patient's admission. Serum cryptococcal antigen test was also positive.

The patient was started on induction therapy with liposomal amphotericin B and flucytosine, which was challenging because of her agitation and the flucytosine availability only as an oral medication. Her CSF fungal culture was clear of Cryptococcus by day 4 of the induction therapy. She sustained a pre-renal acute kidney injury after 10 days of induction therapy, most likely due to amphotericin $B$, and was resuscitated with daily albumin, but the creatinine remained elevated above baseline throughout her admission. Her mental status would wax and wane with LPs and her hospital course was complicated by hospital-acquired pneumonia requiring intubation. Following goals of care discussion with family, she was terminally extubated and died 29 days after initiation of antifungal therapy.

\section{Case 2}

A 57 year-old man presented with shortness of breath due to left hepatic hydrothorax. He had a past medical history of decompensated hepatitis $\mathrm{C}$ virus cirrhosis complicated by refractory ascites status post-transjugular intrahepatic portosystemic shunt placement and $\mathrm{HE}$, and he was listed on the liver transplant waiting list. He also had a history of treated neurosyphilis without documented cure by repeat LP. On review

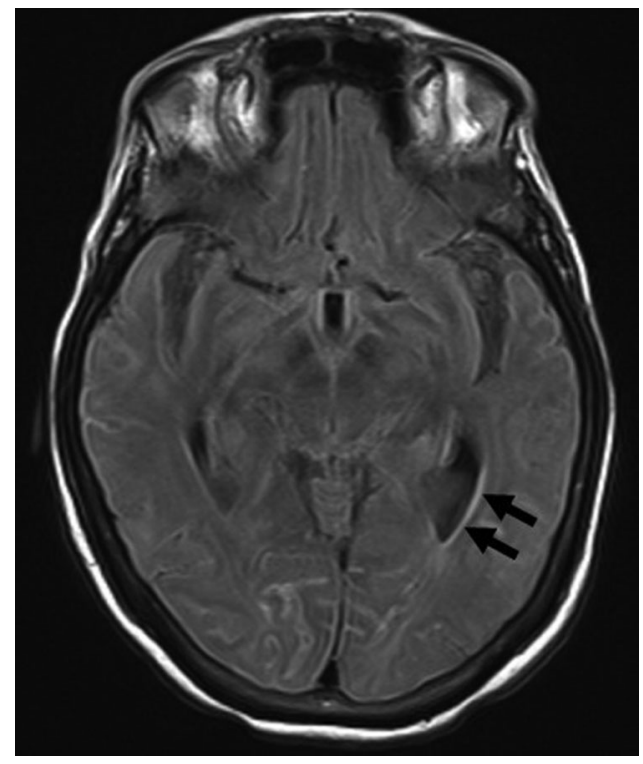

Fig. 1. MRI with and without contrast for Case 1. Curvilinear pial/subarachnoid enhancement and T2/FLAIR hyperintensity in the left temporal lobe is shown adjacent to the left posterior horn (black arrows).

of systems, he described progressive headache, gait disturbance, and hearing loss over the span of months. On neurologic exam, he was alert and oriented with no signs of HE or focal neurologic deficits other than mild auditory deficits bilaterally.

The initial treatment focus was on addressing volume overload in the setting of transjugular intrahepatic portosystemic shunt occlusion, but an LP was performed to evaluate for neurosyphilis treatment response. CSF analysis showed white blood cell count of $128 / \mathrm{mm}^{3}$ (105 monocytes, 23 neutrophils), glucose of $14 \mathrm{mg} / \mathrm{dL}$, protein of $219.9 \mathrm{mg} / \mathrm{dL}$, and venereal disease research laboratory titer being $1: 1$ reactive. Due to the increased white blood cell count with monocytic predominance, low glucose and elevated protein in the CSF, cryptococcal antigen stain was requested as an add-on test and returned reactive. Cryptococcus neoformans also grew in fungal culture.

Subsequent opening pressure was measured at $19 \mathrm{cmH}_{2} \mathrm{O}$ and serial LPs were deferred in the absence of new neurologic symptoms. Serum cryptococcal antigen was also positive. The patient was started on induction therapy with liposomal amphotericin B and flucytosine, in addition to repeat neurosyphilis treatment with IV penicillin G. He completed a 2-week course of each. He was then placed on continued flucytosine and fluconazole maintenance therapy, and his headache resolved on follow-up without any new neurologic complaints or deficits.

\section{Discussion}

The cases presented demonstrate the importance of having a high index of suspicion for cryptococcal meningitis in cirrhosis and the utility of LP when altered mental status or other neurologic complaints are not fully explained by HE. These cases illustrate the wide variety of complaints possible in cryptococcal meningitis, from headache, gait disturbance, and hearing loss to mimicking HE. The previous reports and studies of cryptococcal meningitis in non-HIV infected patients with cirrhosis have been based outside of North America primarily. ${ }^{15-19}$ Our cases and review show that this is certainly not a geographically-limited problem and cryptococcal meningitis in 
this patient population should remain an important differential in North America.

Even with timely diagnosis, adequate treatment remains a tremendous challenge, given the toxicity inherent in the current standard of care with amphotericin B and flucytosine induction. Namely, the nephrotoxicity and myelosuppression of this regimen exacerbates existing hepatorenal syndrome and pancytopenia often already present in patients with decompensated cirrhosis. Fluconazole induced hepatotoxicity can happen, but it is not common and should not prevent patients from being on the indicated lifesaving therapy even in the setting of cirrhosis.

Despite the challenges in treatment, a recent multicenter case series describing liver transplant outcomes in cryptococcosis showed that most patients who end up receiving the transplant survived, with the caveat that only 8 of 39 patients $(20.5 \%)$ who were listed for transplant did eventually undergo liver transplantation. Survival rate was $87.5 \%(7 / 8)$ at 3.5 year follow-up among those transplanted, compared with a 90 -day survival rate of $57.1 \%$ for all patients. Risk factors for mortality include more advanced liver disease (by Child-Pugh or MELD score), requirement for hemodialysis or mechanical ventilation, hypotension/shock, altered mental status and fungemia, whereas protective factors notably include headache. Of note, those pretransplant recipients who were ultimately transplanted underwent a median duration of 43 days on antifungal treatment prior to transplantation and a median of 272 days of antifungal therapy posttransplant. ${ }^{20}$

Selection bias in favor of healthier patients undergoing transplant may partly account for the observed trend towards good outcomes in transplant recipients, but it is important to emphasize that 4 out of the 8 patients transplanted in the aforementioned study had disseminated disease, including 3 with meningitis. These observations suggest cryptococcal infection should not be an absolute exclusion criteria and liver transplantation can remain an option in selected patients with decompensated cirrhosis with cryptococcosis who are able to tolerate antifungal therapy and show clinical improvement. According to the American Society of Transplantation guidelines for cryptococcosis, transplant can be considered in patients who completed induction therapy and all the sites that previously yielded positive cultures are no longer positive. ${ }^{21}$ Fluconazole maintenance therapy should be continued for at least 12 months following induction therapy in transplanted patients, but can be extended based on the level of immunosuppression and any residual symptoms. ${ }^{21}$

\section{Acknowledgments}

The authors would like to acknowledge Dr. Robin Avery, an infectious disease specialist at Johns Hopkins, for helpful suggestions on this manuscript.

\section{Conflict of interest}

The authors have no conflict of interests related to this publication.

\section{Author contributions}

Participated in patient care as a resident physician, conducted the literature review and prepared the manuscript draft (PST), participated in patient care as a clinical gastroenterology and hepatology fellow, conducted the literature review and reviewed the manuscript draft (AA), and participated in patient care as the hepatology attending physician, corresponded with specialist consultants and reviewed the final manuscript (TAW).

\section{References}

[1] Spec A, Panackal A, Williamson P, Bennett JE, Garcia-Diaz J, Ostrander D, et al. Cryptococcus Infection Network in Non-Human Immunodeficiency Virus Cohort (CINCH) Study: Initial Report of Treatment and Outcomes. Open Forum Infect Dis 2016;3:122. doi: 10.1093/ofid/ofw194.35.

[2] Mirza SA, Phelan M, Rimland D, Graviss E, Hamill R, Brandt ME, et al. The changing epidemiology of cryptococcosis: an update from population-based active surveillance in 2 large metropolitan areas, 1992-2000. Clin Infect Dis 2003;36:789-794. doi: 10.1086/368091.

[3] Lin YY, Shiau S, Fang CT. Risk factors for invasive Cryptococcus neoformans diseases: a case-control study. PLoS One 2015;10:e0119090. doi: 10. 1371/journal.pone.0119090.

[4] Chuang YM, Ho YC, Chang HT, Yu CJ, Yang PC, Hsueh PR. Disseminated cryptococcosis in HIV-uninfected patients. Eur J Clin Microbiol Infect Dis 2008;27:307-310. doi: 10.1007/s10096-007-0430-1.

[5] Goldman L, Schafer AI. Goldman-cecil medicine. 25th edition. Philadelphia, PA: Elsevier/Saunders, 2016

[6] Spec A, Raval K, Powderly WG. End-stage liver disease is a strong predictor of early mortality in cryptococcosis. Open Forum Infect Dis 2015;3: ofv197. doi: 10.1093/ofid/ofv197.

[7] Kwon-Chung KJ, Fraser JA, Doering TL, Wang Z, Janbon G, Idnurm A, et al. Cryptococcus neoformans and Cryptococcus gattii, the etiologic agents of cryptococcosis. Cold Spring Harb Perspect Med 2014;4:a019760. doi: 10. 1101/cshperspect.a019760.

[8] Carroll KC, Hobden JA, Miller S, Morse SA, Mietzner TA, Detrick B, et al. Medical Mycology. Jawetz, Melnick, \& Adelberg's Medical Microbiology, 27e. New York, NY: McGraw-Hill Education, 2015.

[9] Syme RM, Bruno TF, Kozel TR, Mody CH. The capsule of Cryptococcus neoformans reduces T-lymphocyte proliferation by reducing phagocytosis, which can be restored with anticapsular antibody. Infect Immun 1999;67:46204627.

[10] Noverr MC, Williamson PR, Fajardo RS, Huffnagle GB. CNLAC1 is required for extrapulmonary dissemination of Cryptococcus neoformans but not pulmonary persistence. Infect Immun 2004;72:1693-1699. doi: 10.1128/IAI.72. 3.1693-1699.2004.

[11] Nosanchuk JD, Casadevall A. The contribution of melanin to microbial pathogenesis. Cell Microbiol 2003;5:203-223. doi: 10.1046/j.1462-5814.2003. 00268.x.

[12] Liu TB, Perlin DS, Xue C. Molecular mechanisms of cryptococcal meningitis. Virulence 2012;3:173-181. doi: 10.4161/viru.18685.

[13] Albillos A, Lario M, Álvarez-Mon M. Cirrhosis-associated immune dysfunction: distinctive features and clinical relevance. J Hepatol 2014;61:13851396. doi: 10.1016/j.jhep.2014.08.010.

[14] Sipeki N, Antal-Szalmas P, Lakatos PL, Papp M. Immune dysfunction in cirrhosis. World J Gastroenterol 2014;20:2564-2577. doi: 10.3748/wjg.v20. i10.2564.

[15] Rubio-Félix SA, Aznar R, Escobedo JA, Mairal P. Cirrhosis and cryptococcal meningitis. Rev Clin Esp 1992;191:396.

[16] Artru P, Schleinitz N, Gauzere BA, Artru S, Paganin F, Roblin X. Neuromeningeal cryptococcosis and alcoholic cirrhosis. Gastroenterol Clin Biol 1997;21: 78-81.

[17] Lee SJ, Choi HK, Son J, Kim KH, Lee SH. Cryptococcal meningitis in patients with or without human immunodeficiency virus: experience in a tertiary hospital. Yonsei Med J 2011;52:482-487. doi: 10.3349/ymj.2011.52.3. 482.

[18] Miranda ÉJ, Gonçalves LG, França FO. Cryptococcal meningitis in HIVnegative patient with liver cirrhosis due to hepatitis C. Braz J Infect Dis 2011;15:399-400. doi: 10.1016/S1413-8670(11)70214-8.

[19] Choi HM, Jung GM, Lee WK, Lee HS, Kim BS, Seong CS, et al. A case of cryptococcal meningitis mimicking hepatic encephalopathy in a patient with liver cirrhosis caused by chronic hepatitis C. Korean J Gastroenterol 2014;64:294-297. doi: 10.4166/kjg.2014.64.5.294.

[20] Singh N, Sifri CD, Silveira FP, Miller R, Gregg KS, Huprikar S, et al. Cryptococcosis in patients with cirrhosis of the liver and posttransplant outcomes. Transplantation 2015;99:2132-2141. doi: 10.1097/TP.0000000000000690.

[21] Baddley JW, Forrest GN. Cryptococcosis in solid organ transplantation. Am J Transplant 2013;13:242-249. doi: 10.1111/ajt.12116. 